\title{
Collective excitations of trapped one-dimensional dipolar quantum gases
}

\author{
P. Pedri, ${ }^{1}$ S. De Palo, ${ }^{2}$ E. Orignac, ${ }^{3}$ R. Citro, ${ }^{4}$ and M. L. Chiofalo ${ }^{5,6}$ \\ ${ }^{1}$ Laboratoire de Physique Théorique e Modèles Statistiques, Université Paris-Sud, Orsay, France \\ ${ }^{2}$ DEMOCRITOS INFM-CNR and Dipartimento di Fisica Teorica, Università Trieste, Trieste, Italy \\ ${ }^{3}$ Laboratoire de Physique de l'École Normale Supérieure de Lyon, CNRS-UMR5672, Lyon, France \\ ${ }^{4}$ Dipartimento di Fisica "E. R. Caianiello" and CNISM, Università degli Studi di Salerno, Salerno, Italy \\ ${ }^{5}$ INFN Dipartimento di Matematica, Facoltà di Farmacia, Università di Pisa, Pisa, Italy \\ ${ }^{6}$ Centre Émile Borel, Institut Henri Poincaré, Paris, France \\ (Received 4 August 2007; published 29 January 2008)
}

\begin{abstract}
We calculate the excitation modes of a one-dimensional (1D) dipolar quantum gas confined in a harmonic trap with frequency $\omega_{0}$ and predict how the frequency of the breathing $n=2$ mode characterizes the interaction strength evolving from the Tonks-Girardeau value $\omega_{2}=2 \omega_{0}$ to the quasi-ordered, superstrongly interacting value $\omega_{2}=\sqrt{5} \omega_{0}$. Our predictions are obtained within a hydrodynamic Luttinger-liquid theory after applying the local density approximation to the equation of state for the homogeneous dipolar gas, which are in turn determined from reptation quantum Monte Carlo simulations. They are shown to be in quite accurate agreement with the results of a sum-rule approach. These effects can be observed in current experiments, revealing the Luttinger-liquid nature of 1D dipolar Bose gases.
\end{abstract}

DOI: 10.1103/PhysRevA.77.015601

PACS number(s): 03.75.Kk, 03.75.Hh, 71.10.Pm, 02.70.Ss

The bottom line in the design of conceptually novel technological applications is the possibility of reaching extreme quantum degeneracy under controlled conditions. This is quite a remarkable property of ultracold atomic gases, which is being evidenced by the interdisciplinary contribution of quantum optics, quantum information, condensed matter, atomic, and fundamental physics. The extreme quantum limit can here be obtained after lowering the temperature down to the nanokelvin scale and by tuning the atomic interactions and the dimensionality. Dimensionality can indeed be reduced to two and one dimensions (1D) after using a variety of magnetic and optical techniques including optical lattices [1-4]. The interactions can also be manipulated almost at will in their short-range part by means of the Fano-Feshbach resonance mechanism [5-9]. In the case of atomic or molecular species with large magnetic or electric moments, proposals have been put forward which predict the possibility of tuning the long-range dipolar tail of the interaction $[10,11]$. The observation of dipolar interactions [12] in atomic ${ }^{52} \mathrm{Cr}$ vapors with relatively large magnetic moment $\mu \simeq 6 \mu_{B}$ is especially promising for applications, since it opens the way to the realization of, e.g., different quantum phases $[13,14]$ and the observation of spin-charge separation [15]. Molecular dipolar crystals have been more recently proposed as a realization of high-fidelity quantum memory for quantum computation [16]. In a set of landmark experiments $[17,18]$ and supported by simulational work $[19,20]$, vapors of ${ }^{52} \mathrm{Cr}$ atoms have been Bose condensed [21]. Yet the effect of the dipolar interaction has been largely enhanced after reducing the strength of the short-range part [22].

The combination of 1D geometries with tunable interactions may provide an easy access to enhanced quantum correlations. Experimental realizations of the strongly correlated Tonks-Girardeau (TG) gas [23] have already been observed in atomic vapors with contact interactions [24,25]. Going beyond the TG regime under these conditions is not an easy task [26]. However, as we have more recently demonstrated by a combined reptation quantum Monte Carlo (RQMC) and bosonization approach [27], a homogeneous 1D Bose gas with dipolar interactions is a very strongly correlated Luttinger liquid with the parameter $K<1$ [28] at all densities $n r_{0} \gtrsim 0.1$ with $r_{0}$ being the range of the dipolar potential (see below). The Luttinger liquid crosses over from a TonksGirardeau gas setting in for $n r_{0} \lesssim 0.1$ to a high-density quasiordered state [29] which can be viewed as the analog of a charge density wave. We have also discussed how the use of polar molecules may provide access to this quasi-ordered state, which from now on we refer to as a dipolar density wave (DDW).

Knowledge of the interaction regime is a basic tool for all conceivable applications. Since ongoing experiments will be performed in confined geometry, one of the best suited and controlled methods is to exploit the collective excitations related to the discrete modes in the harmonic trap [30], which has indeed been largely used since the very first experiments [31,32]. The study of the collective modes can also be useful in view of the proposal to implement the quantum memory, to reveal and investigate decoherence mechanisms possibly arising from the coupling of internal and external degrees of freedom on the DDW state [16].

In this Brief Report we predict the crossover behavior of the collective modes of the trapped 1D dipolar Bose gas by two different theoretical methods: namely, a sum-rule approach and a hydrodynamic Luttinger-liquid model. The two methods share the application of the local density approximation (LDA) to the equation of state as determined from our RQMC simulational data and are shown to give results in quite remarkable agreement.

We first determine the ground-state energy per particle (within the statistical error) of the homogeneous dipolar Bose gas by resorting to RQMC simulations [33]. As described in more detail in Ref. [27], we consider $N$ atoms or molecules of mass $M$ and permanent dipole moments arranged along a line in the limit of negligible contact interaction [22] and polarized in the orthogonal direction. The 
Hamiltonian $H=\left[-\left(n r_{0}\right)^{2} \Sigma_{i}\left(\partial^{2} / \partial x_{i}^{2}\right)+\left(n r_{0}\right)^{3} \Sigma_{i<j}\left|x_{i}-x_{j}\right|^{-3}\right]$ is defined in effective Rydberg units $R y^{*}=\hbar^{2} /\left(2 M r_{0}^{2}\right)$ with $r_{0} \equiv M C_{d d} /\left(2 \pi \hbar^{2}\right)$ the effective Bohr radius, $C_{d d}=\mu_{0} \mu_{d}^{2}$ or $C_{d d}=d^{2} / \epsilon_{0}$ the interaction strengths for magnetic $\mu_{d}$ or electric $d$ dipole moments, respectively. The governing dimensionless parameter is $n r_{0}$, with $n$ the number of dipoles per unit length.

The RQMC data for the dependence of the energy per particle $\varepsilon$ on $n$ are fitted by the expression

$$
\begin{aligned}
\varepsilon\left(n r_{0}\right) / R y^{*}= & {\left[\zeta(3)\left(n r_{0}\right)^{4}+a\left(n r_{0}\right)^{e}+b\left(n r_{0}\right)^{f}+c\left(n r_{0}\right)^{2+g}\right] } \\
& \times\left[1+n r_{0}\right]^{-1}+\left(\pi^{2} / 3\right)\left(n r_{0}\right)^{2}\left[1+d\left(n r_{0}\right)^{g}\right]^{-1},
\end{aligned}
$$

where $\quad a=3.1(1), \quad b=3.2(2), \quad c=4.3(4), \quad d=1.7(1)$, $e=3.503(4), f=3.05(5)$, and $g=0.34(4)$ with an overall $\chi_{\text {red }}^{2} \simeq 5$. For $n r_{0} \ll 1, \varepsilon\left(n r_{0}\right) / R y^{*} \sim\left(\pi^{2} / 3\right)\left(n r_{0}\right)^{2}$, and for $n r_{0} \gg 1, \quad \varepsilon\left(n r_{0}\right) / R y^{*} \sim \zeta(3)\left(n r_{0}\right)^{3}$ so that both the TG and DDW limits are satisfied. The functional form for $\varepsilon(n)$ here provided can be used in further calculations. Here, we use it to obtain the chemical potential $\mu_{\mathrm{RQMC}}(n)$ $=[1+n(\partial / \partial n)] \varepsilon(n)$.

In experiments, the effectively 1D dipolar gas is confined by a harmonic potential $V(x)=M \omega_{0}^{2} x^{2} / 2$ in the axial direction. The dynamical behavior of the confined gas can be found from hydrodynamic equations at temperature $T=0$ using a LDA to the equation of state, in which the energy of the inhomogeneous system is a local functional of the local density $n(x)$ expressed as the integral over $x$ of the energy density of the homogeneous gas with density $n(x)$. The validity of such an approach is limited to dynamical effects in which the typical length over which $n(x)$ varies is much larger than the average interparticle distance in the axial direction. Furthermore, the dynamical behavior in the radial (transverse) plane must remain frozen. Under these conditions, the ground-state density profile $n(x)$ of the trapped dipolar gas is obtained by plugging $\mu_{\mathrm{RQMC}}(n)$ into the equation

$$
\mu(n(x))+V(x)=\mu_{0}
$$

and solving it for $\mu_{0}$ and $n(x)$ with the condition that the total number of particles $N=\int_{-R}^{R} n(x) d x$ be conserved, where the Thomas-Fermi radius $R=\left[2 \mu_{0} /\left(M \omega_{0}^{2}\right)\right]^{1 / 2}$ is such that $n( \pm R)=0$. The condition can be cast in the form $N\left(r_{0} / a_{\mathrm{ho}}\right)^{2}=\left(\mu_{0} / R y^{*}\right)^{1 / 2} \int_{-1}^{1} r_{0} \mu^{-1}\left[\left(\mu_{0} / R y^{*}\right)\left(1-x^{2}\right)\right] d x$, where $a_{\mathrm{ho}}=\left(\hbar / M \omega_{0}\right)^{1 / 2}$ is the harmonic oscillator length. This identifies $N\left(r_{0} / a_{\mathrm{ho}}\right)^{2}$ as the interaction parameter driving the trapped dipolar gas from the TG across the DDW regime.

The evolution of the calculated density profiles through the crossover shows the expected increase of the central density $n(0)$ with the chemical potential $\mu_{0}$, as well as a steepening of the profiles at the trap edges. The calculated profiles agree with the analytical results expected in the TG and DDW limits.

We first determine the effect of the interactions on the frequency of the lowest compressional (breathing) mode of the trapped gas by a sum-rule approach. This mode is coupled to the ground state by the operator $\hat{X}_{2}=\sum_{i=1}^{N} x_{i}^{2}$. This

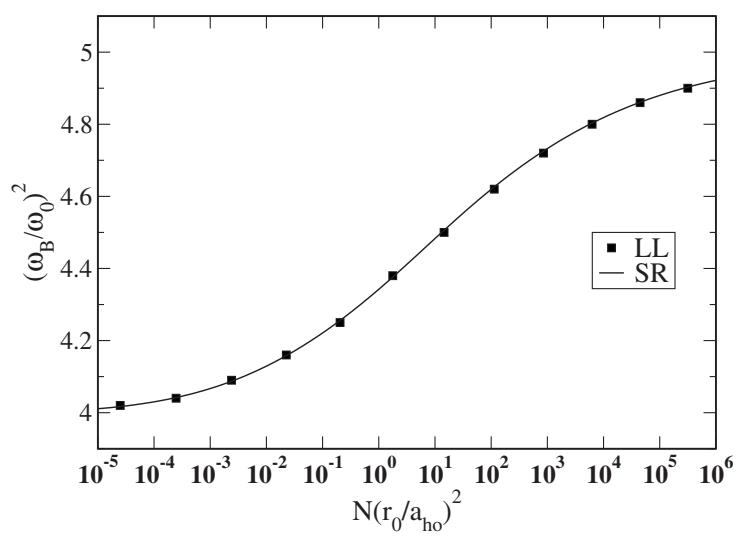

FIG. 1. Squared frequency $\omega_{B}^{2}$ of the breathing mode scaled to the trap frequency $\omega_{0}$ vs the interaction parameter $N\left(r_{0} / a_{h o}\right)^{2}$, as calculated from two models. Solid line: sum-rule approach, Eq. (2) (SR). Symbols: Luttinger-liquid hydrodynamics, Eq. (6) (LL).

makes it the easiest to probe in current experiments, as is excited by modulating the trap frequency and observed by following the time evolution of the width of the cloud by conventional density-imaging techniques. The frequency $\omega_{B}$ of the breathing mode satisfies an inequality that can be derived from the sum rule [34-36] $\omega_{B}^{2} \leq m_{1} / m_{-1}$, where $2 m_{1}=\left\langle\left[X_{2},\left[H, X_{2}\right]\right]\right\rangle$ and $2 m_{-1}$ is the static response function. After some simplification, this yields the upper bound

$$
\omega_{B}^{2} \leq \Omega_{B}^{2}=-2 \frac{\left\langle x^{2}\right\rangle}{\partial\left\langle x^{2}\right\rangle / \partial \omega_{0}^{2}},
$$

where $\left\langle x^{2}\right\rangle=N^{-1} \int x^{2} n(x) d x$. A closed-form expression of $\Omega_{B}$ in Eq. (2) can be obtained by means of a scaling argument whenever $\mu(n)$ is of the form $\mu(n)=\lambda n^{\gamma}$, resulting in $\Omega_{B}=\omega_{0}(2+\gamma)^{1 / 2}$. In particular, in the TG case with $\gamma=2$ this yields $\Omega_{B}=2 \omega_{0}$, and in the DDW case with $\gamma=3$ it yields $\Omega_{B}=\sqrt{5} \omega_{0}$.

For intermediate interaction strengths, we have to resort to a numerical estimation of Eq. (2) using the LDA density profile. The result is represented by the solid line in Fig. 1, showing the smooth evolution of the breathing-mode frequency $\omega_{B}$ from the TG to the DDW regimes. Figure 1 represents a useful tool to identify the interaction regime of the trapped dipolar gas through one of the best handled experimental probes available with cold atomic (molecular) quantum gases [31,32]. However, Eq. (2) in principle yields an upper bound for the frequency of the lowest compressional mode. Moreover, estimating the frequencies of the higher modes by the sum-rule approach is not as simple. We thus switch to an alternative, hydrodynamic, approach to compute their frequencies.

In [27], we have shown that the low-energy behavior of a homogeneous dipolar Bose gas is well described by the Luttinger Hamiltonian, and we have determined the density dependence of the velocity $u$ and Luttinger exponent $K$ by combining bosonization and RQMC techniques. The $u$ and $K$ obtained from the RQMC structure factor were found to agree with those extracted from the RQMC energy via the 
Luttinger relations $u K=M^{-1} \pi n$ and $u / K=\pi^{-1} \partial_{n} \mu(n)$ embodying Galilean invariance $[37,38]$. We now assume that in a slowly varying external trapping potential, the dipolar Bose gas can be described by a Luttinger liquid Hamiltonian

$$
H_{\mathrm{LL}}=\int_{-R}^{R} \frac{d x}{2 \pi}\left[u(x) K(x)(\pi \Pi)^{2}+\frac{u(x)}{K(x)}\left(\partial_{x} \phi\right)^{2}\right],
$$

where $u(x)$ and $K(x)$ now depend on position via the LDA $n(x)$. They are related through $u(x) K(x)=M^{-1} \pi n(x)$ and $u(x) / K(x)=\left.\pi^{-1} \partial_{n} \mu(n)\right|_{n=n(x)}$, extending the Luttinger-liquid relations to the weakly inhomogeneous system. In Eq. (3), $\phi$ and $\Pi$ satisfy canonical commutation relations $[\phi(x), \Pi(y)]$ $=i \delta(x-y)$, leading to the equations of motion

$$
\begin{gathered}
\partial_{t} \phi(x, t)=\pi u(x) K(x) \Pi(x, t), \\
\pi \partial_{t} \Pi(x, t)=\partial_{x}\left(\frac{u(x)}{K(x)} \partial_{x} \phi(x, t)\right) .
\end{gathered}
$$

These equations of motion must be complemented by boundary conditions expressing that no current flows across the edges-i.e., $j( \pm R, t)=0$. Since $\delta n=-\partial_{x} \phi / \pi$, the continuity equation $\partial_{t}(\delta n)+\partial_{x} j=0$ leads naturally to $j=\partial_{t} \phi / \pi$ and allows us to rewrite the boundary conditions as $\phi(-R)=\phi_{0}$ and $\phi(R)=\phi_{1}[39]$.

Equations (4) and (5) possess a stationary solution $\partial_{x} \phi \propto K(x) / u(x)$. This solution actually describes the addition of one particle to the system $\phi(x) \rightarrow \phi(x)-\pi g(x) / g(R)$ where $g(x)=\int_{-R}^{x}[K(y) / u(y)] d y$. A consequence is that $\phi_{0}$ and $\phi_{1}$ are not independent, but related through $\phi_{1}-\phi_{0}=-\pi N$ with $N$ being the number of particles added to the system. This form generalizes the bosonization formula derived in the case of a homogeneous system [37]. Alternatively, the static solution can be derived by considering the effect of a perturbation to the trapping potential [40]. Combining Eqs. (4) and (5) and using linearity to search for solutions of the form $\phi(x, t)=\phi_{0}-\pi N g(x) / g(R)+\sum_{n \geq 1} \varphi_{n}(x) e^{i \omega_{n} t}$ we find

$$
-M \omega_{n}^{2} \varphi_{n}=n(x) \partial_{x}\left[\partial_{n} \mu(n(x)) \partial_{x} \varphi_{n}\right],
$$

with the boundary conditions $\varphi_{n}( \pm R)=0$ for the discrete Fourier components $\varphi_{n}$.

While Eq. (6) is cast in a form identical to the hydrodynamic equation for density excitations [36], we remark that here it has been derived from $H_{\mathrm{LL}}$, Eq. (3). Thus, a comparison of the measured excitation frequencies with those predicted from (6) with our RQMC data provides a test of Luttinger-liquid behavior in trapped 1D dipolar Bose gases within the validity of the LDA.

In the case of a harmonic trapping, one of the eigenfrequencies in Eq. (6) is obtained straightforwardly. Indeed, substituting $\varphi_{1}(x) \propto n(x)$ into Eq. (6), differentiating Eq. (1) with respect to $x$, and using the Luttinger-liquid relations, we find that $\varphi_{1}(x)$ is an eigenfunction of (6) associated with the eigenvalue $\omega_{0}^{2}$. This particular solution is simply the Kohn mode or sloshing mode describing a center-of-mass oscillation. Indeed, it can be recovered by expanding to first order in $A$ the expression $\phi(x, t)=\phi\left(x-A \cos \left(\omega_{0} t\right), 0\right)$ which describes a center-of-mass motion with rigid density. The

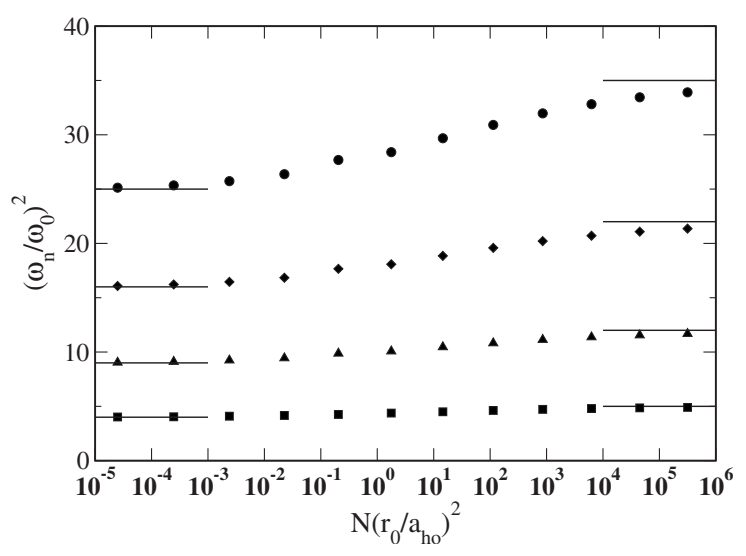

FIG. 2. $\left(\omega_{n} / \omega_{0}\right)^{2}$ vs $N\left(r_{0} / a_{a h o}\right)^{2}$ from Eq. (6). From bottom to top: the modes with $n=2,3,4$, and 5 . Tick solid lines: limiting values in the TG and DDW regimes at each $n$.

eigenfrequencies are exactly known also in the two asymptotic limits. Indeed, insertion of $\mu(n) \propto n^{\gamma}$ into Eqs. (1) and (6) yields solutions of the form $\varphi_{n}(x)$ $=A_{n}\left(1-x^{2} / R^{2}\right)^{1 / \gamma} C_{n}^{(1 / \gamma+1 / 2)}(x / R)$, the associated eigenvalues being $\omega_{n}^{2}=\omega_{0}^{2} n[2+(n-1) \gamma] / 2[36,40]$, where $C_{n}^{(\alpha)}$ are Gegenbauer polynomials and $A_{n}$ normalization factors [41]. Thus, at the two opposite TG $(\gamma=2)$ and DDW $(\gamma=3)$ limits one finds, respectively, $\omega_{n}=n^{2} \omega_{0}^{2}$ and $\omega_{n}=n(3 n-1) \omega_{0}^{2} / 2$. For intermediate densities, we have solved Eq. (6) using the SLEDGE algorithm available online [42], after inserting as ingredients the computed LDA density profiles from Eq. (1) and the analytical expression for $\partial_{n} \mu$ obtained from the RQMC fit, evaluated at the local density for different values of the interaction parameter $N\left(r_{0} / a_{\mathrm{ho}}\right)^{2}$. In the numerical solution we have taken special care of the finite-mesh-size effects for best accuracy.

The values of the breathing frequency $\omega_{B} / \omega_{0}$ obtained from Eq. (6) are represented in Fig. 1 by the solid symbols and agree up to the second digit with the sum-rule result. This was expected in the TG and DDW regimes where, as already noticed in Ref. [36], an equality sign holds in Eq. (2). The eigenfrequencies of the higher modes with $n=3,4$, and 5 are displayed in Fig. 2 as functions of $N\left(r_{0} / a_{\mathrm{ho}}\right)^{2}$, showing the same smooth crossover behavior between the two opposite TG and DDW regimes. The exact frequencies in these asymptotic regimes are recovered by the numerical calculations.

In conclusion, we have predicted the evolution of the collective modes of a 1D dipolar Bose gas through the crossover from the Tonks-Girardeau to the dipolar-density-wave regime. These modes and especially the breathing mode can be excited and measured by quite standard and reliable techniques. Our results are relevant to experiments, where they can be used to determine the interaction regime and extended to investigate the occurrence of decoherence effects in quantum applications [16]. From the theoretical point of view, our results confirm for the trapped gas the smooth crossover from the TG to the DDW regimes [27], as expected for weak 
inhomogeneity. The RQMC functional form of the energy per particle that we explicitly provide in this work can be useful to investigate the issue, crucial for applications, of the crystal phase stabilization by means of, e.g., a commensurate, though shallow, optical lattice.
M.L.C. would like to thank the Institut Henri PoincaréCentre Emile Borel in Paris for hospitality and support. This work was supported by the Ministère de la Recherche (Grant No. ACI Nanoscience 201), by the ANR (Grants Nos. NT0542103 and 05-Nano-008-02), and by the IFRAF Institute.
[1] D. Hellweg et al., Appl. Phys. B: Lasers Opt. 73, 781 (2001).

[2] A. Görlitz et al., Phys. Rev. Lett. 87, 130402 (2001).

[3] I. Bloch, Nat. Phys. 1, 23 (2005) and references therein.

[4] S. Richard et al., Phys. Rev. Lett. 91, 010405 (2003).

[5] H. Feshbach, Ann. Phys. 5, 357 (1958).

[6] U. Fano, Phys. Rev. 124, 1866 (1961).

[7] S. Inouye et al., Nature (London) 392, 151 (1998).

[8] J. L. Roberts et al., Phys. Rev. Lett. 86, 4211 (2001).

[9] T. Weber, J. Herbig, M. Mark, H.-C. Nägerl, and R. Grimm, Science 299, 232 (2003).

[10] S. Giovanazzi, A. Görlitz, and T. Pfau, Phys. Rev. Lett. 89, 130401 (2002).

[11] H. P. Büchler et al., Phys. Rev. Lett. 98, 060404 (2007).

[12] J. Stuhler et al., Phys. Rev. Lett. 95, 150406 (2005).

[13] K. Góral, L. Santos, and M. Lewenstein, Phys. Rev. Lett. 88, 170406 (2002).

[14] H. Pu, W. Zhang, and P. Meystre, Phys. Rev. Lett. 87, 140405 (2001).

[15] A. Kleine, C. Kollath, I. McCulloch, T. Giamarchi, and U. Schollwoeck, e-print arXiv:0706.0709.

[16] P. Rabl and P. Zoller, Phys. Rev. A 76, 042308 (2007).

[17] L. Santos, G. V. Shlyapnikov, P. Zoller, and M. Lewenstein, Phys. Rev. Lett. 85, 1791 (2000).

[18] K. Góral, K. Rzażewski, and T. Pfau, Phys. Rev. A 61, 051601(R) (2000).

[19] K. Nho and D. P. Landau, Phys. Rev. A 72, 023615 (2005).

[20] S. Ronen, D. C. E. Bortolotti, D. Blume, and J. L. Bohn, Phys. Rev. A 74, 033611 (2006).

[21] A. Griesmaier, J. Werner, S. Hensler, J. Stuhler, and T. Pfau, Phys. Rev. Lett. 94, 160401 (2005).

[22] T. Lahaye et al., Nature (London) (to be published).

[23] M. Girardeau, J. Math. Phys. 1, 516 (1960).

[24] B. Paredes et al., Nature (London) 429, 277 (2004).
[25] T. Kinoshita, T. Wenger, and D. S. Weiss, Science 305, 1125 (2004).

[26] G. E. Astrakharchik, J. Boronat, J. Casulleras, and S. Giorgini, Phys. Rev. Lett. 95, 190407 (2005).

[27] R. Citro, E. Orignac, S. De Palo, and M. L. Chiofalo, Phys. Rev. A 75, 051602(R) (2007).

[28] T. Giamarchi, Quantum Physics in One Dimension (Oxford University Press, Oxford, 2004).

[29] A. S. Arkhipov, G. E. Astrakharchik, A. V. Belikov, and Y. E. Lozovik, JETP Lett. 82, 39 (2005).

[30] S. Stringari, Phys. Rev. Lett. 77, 2360 (1996).

[31] D. S. Jin, M. R. Matthews, J. R. Ensher, C. E. Wieman, and E. A. Cornell, Phys. Rev. Lett. 78, 764 (1997).

[32] D. M. Stamper-Kurn, H. J. Miesner, S. Inouye, M. R. Andrews, and W. Ketterle, Phys. Rev. Lett. 81, 500 (1998).

[33] S. Baroni and S. Moroni, Phys. Rev. Lett. 82, 4745 (1999).

[34] L. P. Pitaevskii and S. Stringari, Bose-Einstein Condensation (Oxford University Press, New York, 2003).

[35] M. L. Chiofalo, S. Conti, and M. P. Tosi, J. Phys.: Condens. Matter 8, 1921 (1996).

[36] C. Menotti and S. Stringari, Phys. Rev. A 66, 043610 (2002).

[37] F. D. M. Haldane, Phys. Rev. Lett. 47, 1840 (1981).

[38] T. Giamarchi, Quantum Physics in One Dimension (Oxford University Press, Oxford, 2004).

[39] L. Kecke, H. Grabert, and W. Hausler, Phys. Rev. Lett. 94, 176802 (2005).

[40] R. Citro et al. (unpublished).

[41] D. Petrov, D. Gangardt, and G. Shlyapnikov, J. Phys. IV 116, 3 (2004).

[42] S. Pruess and C. Fulton, ACM Trans. Math. Softw. 19, 360 (1993). See also the website http://www.mines.edu/ fs_home/spruess/. 